IZA DP No. 6031

Inference for Shared-Frailty Survival Models with Left-Truncated Data

Gerard J. van den Berg

Bettina Drepper

October 2011 


\title{
Inference for Shared-Frailty Survival Models with Left-Truncated Data
}

\author{
Gerard J. van den Berg \\ University of Mannheim, VU University Amsterdam, \\ IFAU-Uppsala and IZA \\ Bettina Drepper \\ University of Mannheim
}

\section{Discussion Paper No. 6031 \\ October 2011}

\author{
IZA \\ P.O. Box 7240 \\ 53072 Bonn \\ Germany \\ Phone: +49-228-3894-0 \\ Fax: +49-228-3894-180 \\ E-mail: iza@iza.org
}

Any opinions expressed here are those of the author(s) and not those of IZA. Research published in this series may include views on policy, but the institute itself takes no institutional policy positions.

The Institute for the Study of Labor (IZA) in Bonn is a local and virtual international research center and a place of communication between science, politics and business. IZA is an independent nonprofit organization supported by Deutsche Post Foundation. The center is associated with the University of Bonn and offers a stimulating research environment through its international network, workshops and conferences, data service, project support, research visits and doctoral program. IZA engages in (i) original and internationally competitive research in all fields of labor economics, (ii) development of policy concepts, and (iii) dissemination of research results and concepts to the interested public.

IZA Discussion Papers often represent preliminary work and are circulated to encourage discussion. Citation of such a paper should account for its provisional character. A revised version may be available directly from the author. 
IZA Discussion Paper No. 6031

October 2011

\section{ABSTRACT \\ Inference for Shared-Frailty Survival Models with Left-Truncated Data*}

Shared-frailty survival models specify that systematic unobserved determinants of duration outcomes are identical within groups of individuals. We consider random-effects likelihoodbased statistical inference if the duration data are subject to left-truncation. Such inference with left-truncated data can be performed in the Stata software package. We show that with left-truncated data, the commands ignore the weeding-out process before the left-truncation points, affecting the distribution of unobserved determinants among group members in the data, that is, among the group members who survive until their truncation points. We critically examine studies in the statistical literature on this issue as well as published empirical studies that use the commands. Simulations illustrate the size of the (asymptotic) bias and its dependence on the degree of truncation. We provide a Stata command file that maximizes the likelihood function that properly takes account of the interplay between truncation and dynamic selection.

JEL Classification: C41, C34

Keywords: $\quad$ stata, duration analysis, left-truncation, likelihood function, dynamic selection, hazard rate, unobserved heterogeneity, twin data

Corresponding author:

Gerard J. van den Berg

Department of Economics

University of Mannheim

L7, 3-5

68131 Mannheim

Germany

E-mail: gerard@uni-mannheim.de

* We thank Arne Uhlendorff for helpful comments. Stata is a registered trademark of StataCorp LP. 


\section{Introduction}

In this paper we consider inference for shared-frailty survival models. These are Mixed Proportional Hazard (MPH) models in which systematic unobserved determinants of duration outcomes are identical within units or groups of individuals. We allow the spell durations to be subject to left-truncation, meaning that the duration outcome is only observed if it exceeds a certain threshold value, and we focus on random-effects likelihood-based inference. We show that the Stata software package command to estimate shared-frailty survival models in the presence of left-truncated duration data should not be applied, since it maximizes a likelihood function that does not properly take account of dynamic selection before the truncation points.

In order to explain this and to motivate the relevance of our contribution, we start with an introduction into the survival models with unobserved heterogeneity (or frailty terms) that are included in Stata for statistical inference. Shared-frailty models are an important class of such models.

Empirical survival studies or studies in duration analysis commonly adopt some version of the Mixed Proportional Hazard (MPH) model for the hazard rate. The MPH model stipulates that the individual hazard rate (or exit rate out of the current state) $\theta$ depends on the elapsed duration $t$, on explanatory variables $x$ and on unobserved determinants $v$ such that

$$
\theta(t \mid x, v)=\lambda(t) \phi(x) v
$$

at all $t, x, v$ for some functions $\lambda$ and $\phi$ (see Lancaster, 1990, and Van den Berg, 2001 , for surveys). Here, $\phi$ is the function of interest although sometimes $\lambda$ is also of interest. Typically, at least some elements of the vector $x$ are time-varying, but for ease of exposition we ignore this in this paper. Notice that without loss of generality $v$ can be seen as the joint multiplicative effect of a vector of unobserved determinants on the individual hazard rate. The term $v$ is often called the frailty term. It is not directly estimated from the data, as it varies across individuals. Moreover, in contrast to linear regression analysis, ignoring unobserved heterogeneity leads to biased estimates of $\lambda$ and $\phi$. This is because individuals with a high $v$ leave the state of interest on average earlier than individuals with low $v$. This phenomenon is called "weeding out" or "sorting". It may occur at different speeds for different $x$, causing the composition of survivors in terms of $v$ to change over time. In general, ignoring this leads to a negative bias in the estimate of $\lambda(t)$ and a bias in the estimated covariate effects (Lancaster, 1990, Van den Berg, 2001). The most common approach for inference is to assume that $v$ has a 
distribution $G$ in the population and to estimate its parameters along with (the parameters of) $\lambda$ and $\phi$ using Maximum Likelihood Estimation, where the likelihood contribution of an individual spell integrates over $G$. In econometrics, this is called random-effects estimation. To ensure that identification is not fully driven by functional form assumptions, it is assumed that $x$ and $v$ are independently distributed in the population and that $\mathrm{E}(v)=1$. The population constitutes the inflow into the state of interest (although this may be modified; see below). By far the most common functional form for $G$ is the gamma distribution. This can be justified as an approximation to a wide class of frailty distributions (Abbring and Van den Berg, 2007). The approximation improves with left-truncation of the durations. An alternative frailty distribution is the Inverse-Gaussian distribution.

Often it is natural to assume that small subsets of different individuals or spell durations share the same value of $v$. For example, different unemployment spells of the same person may share the same unobserved determinant $v$. Or the mortality rates of identical twins may assumed to depend on identical unobserved determinants $v$. In general, the data may identify groups or units or strata such that different spells within a group or unit or stratum share the same $v$. Data with this feature are often called multi-spell duration data. To keep the terminology simple, consider the case where for each unit in the sample we observe at most two spells. The unit has a given value of $v$, and we assume that its spell durations are independent drawings from the univariate duration distribution of $t$ given $x, v$, where, of course, $v$ is unobserved, so that the durations given $x$ are not independent. It depends on the context whether $x$ is also identical across spells or individuals within a unit. For ease of exposition, we take the data to consist of a random sample of units. We return to this below.

The multi-spell MPH model was first proposed by Clayton (1978) and is nowadays known under the name "shared-frailty model". Notice that it has the same unknown functions as the single-spell MPH model, namely $\lambda, \phi$ and $G$. The empirical analysis of shared-frailty models is widespread (see e.g. Hougaard, 2000, and Van den Berg, 2001, for surveys). If the underlying modeling assumptions are correct, multi-spell data enable identification of the MPH model under weaker assumptions than single-spell data, and the estimation results are more robust with respect to functional-form assumptions (Van den Berg, 2001). By straightforward extension of the estimation with single-spell data, the most common estimation methods are random-effect procedures where each unit or group provides a likelihood contribution that integrates over the distribution $G$ of $v$ across the units and where $\lambda, \phi$ and $G$ are parameterized. ${ }^{1}$

\footnotetext{
${ }^{1}$ If different individuals within a unit or group have different values of $x$ then Stratified
} 
The Stata software package offers a large number of pre-programmed estimation routines for survival analysis. In this sense Stata is unique among the available software packages covering survival analysis, and indeed it has become popular among empirical researchers. The main survival model estimation command streg also captures the shared-frailty model, by invoking the option shared() to indicate which individuals share the same value of $v$. Gutierrez (2002) gives an overview of parametric shared-frailty models in Stata. See Hirsch and Wienke (2011) for an overview of software packages with estimation routines for sharedfrailty models.

Sampling schemes where durations are left-truncated are common in singlespell as well as in multi-spell survival analysis (Guo, 1993). For example, unemployment duration spells are often only recorded in register data if the duration exceeds one month. Population register data typically follow individuals from a given point in calendar time onwards, where the starting points of the spells that are ongoing at the beginning of the register's observation window are often observed as well. The spells that started say $t_{0}$ time units before the beginning of the observation window are then only observed if the duration exceeds $t_{0}$. With the increasing availability of such register data in socio-economic and health research, the usage of left-truncated duration data has increased. This also applies to multispell data. For example, death causes of Danish twins were only systematically recorded as of January 1, 1943, so to study death causes among those born before 1943, it makes sense to restrict attention to both twin members being alive on January $1,1943 .^{2}$ If the duration from birth until death due to a specific death cause is the relevant duration variable then this variable is left-truncated at the age attained on January 1, 1943. Hence, the left-truncation points as measured in the age dimension differ across twin pairs. In studies with hospital patients, only the patients are observed who survive up to the point when the trial period at the hospital starts. If the patient subsequently experiences remission and relapse then subsequent illness spells may not be left-truncated.

Stata allows for left-truncation of the duration data, through the enter() option when declaring the data as duration data by the stset command. Importantly, the value $t_{0}$ of the truncation threshold may differ across individuals (as

Partial Likelihood Estimation can be used as an alternative (fixed effects) method (Kalbfleisch and Prentice, 1980, Chamberlain, 1985, Ridder and Tunalı, 1999). In the present paper we are not concerned with that method.

${ }^{2}$ After all, if a twin member is observed to have died before 1943 then it is not known whether this was due to the cause of interest or due to another cause. In the latter case, the moment of death due to the cause of interest is right-censored by an event with an unknown distribution, and inference would include the estimation of this distribution. 
well as across spells for a given unit in the case of the shared-frailty model).

Notice that left-truncation gives rise to a second selection issue, on top of the selection generated by the dynamic weeding-out. After all, surviving up to some threshold value is more likely if the frailty term is small. The Stata routine for shared-frailty models ${ }^{3}$ ignores the fact that the second selection impacts on the first selection. Restricting the outcome to exceed a lower threshold implies that the frailty distribution in the sample systematically differs from that in the population upon inflow into the state of interest. ${ }^{4}$ If the former distribution is nevertheless assumed to equal the latter, then, as we shall see, the resulting estimators of $\beta$ and $\lambda$ are inconsistent. One may redefine the population to be the survivors at $t_{0}$ but this only makes sense if $t_{0}$ is identical across all units and spells.

The interplay between left-truncation and dynamic selection has always been recognized in the single-spell survival analysis literature. As we discuss below, with multiple spells the role of this interplay has been obscured. However, we are not the first to point out the importance of dealing with the above interplay and its implication for the frailty distribution in the sample, in shared frailty models. Jensen et al. (2004) provide a lucid account. They contrast the correct likelihood function to the likelihood function where the interplay is ignored for the case of gamma-distributed frailties, and they discuss the bias when using the latter. They point out that Nielsen et al. (1992), which is a seminal paper in survival analysis, used the latter likelihood in the case of left-truncated data in the shared frailty model. Elsewhere in the literature, Rondeau and Gonzalez (2005) use the correct likelihood for their semi-parametric estimator of the shared frailty model in the case of left-truncated data, whereas Do and Ma (2010) use the other likelihood function for their semi-parametric estimator in the same setting.

The remainder of the paper is structured as follows. In Section 2 we discuss left-truncation in multiple spell duration data in more detail. We show under which conditions the likelihood function of the streg, shared() command is misspecified for left-truncated data, and we present the correct likelihood function. We also discuss the analogous problem with the stcox command in Stata for the semi-parametric estimation of the shared gamma frailty model. We list a number of empirical studies that have used this stcox command to estimate shared-frailty models with left-truncated data. In Section 3 we demonstrate in a short simulation study how the magnitude of the bias resulting from the misspecification

\footnotetext{
${ }^{3}$ This routine is available since Version 7, up to and including the current Version 12.

${ }^{4}$ See Ridder (1984) for an account of the differences between frailty distributions in different types of single-spell samples.
} 
depends on the level of truncation and the variance of the frailty distribution. We also examine the performance of the stcox command in this setting, and we list published articles that use this Stata command to semi-parametrically estimate the shared gamma frailty model with left-truncated data. Section 4 concludes. In the Appendix we introduce a corrected Stata command called stregshared.

\section{Likelihood specification with left-truncated du- ration data and shared frailties}

Consider a random sample of single spells, if the MPH model applies. The random sample consists of independent draws from the distribution of $T \mid X$ for various values $x$ of $X$, where $T$ denotes the random duration variable. We consider likelihood-based inference, and for the moment we take $\lambda, \phi$ and $G$ to be parametric functions. The spell durations may be independently right-censored but we are not concerned with that here. Consequently, the likelihood contribution of a single spell is the probability density function $f_{u}(t \mid x)$ of $T \mid X$ evaluated at the observation $(t, x)$, with

$$
f_{u}(t \mid x)=\mathbb{E}_{v}\left(f_{c}(t \mid x, v)\right)=\int_{v} \lambda(t) \phi(x) v \exp (-\Lambda(t) \phi(x) v) d G(v)
$$

in which $\Lambda(t):=\int_{0}^{t} \lambda(u) d u$ denotes the so-called integrated baseline hazard and $f_{c}$ is the probability density function of $T \mid X, V$.

Next, consider a random sample of units each with $j=1,2$ spells that share their frailty term $v$. Throughout the paper we assume that conditional on $v$, the spells are independent. The likelihood contribution of a unit with non-truncated uncensored duration outcomes $t_{1} \mid x_{1}$ and $t_{2} \mid x_{2}$ then equals $\int_{v} f_{c}\left(t_{1} \mid x_{1}, v\right) f_{c}\left(t_{2} \mid x_{2}, v\right) d G(v)$.

Left-truncation of a single-spell duration outcome variable means that the variable is only observed if its value exceeds a lower threshold, say $t_{0}$. Throughout the paper we are only concerned with deterministic $t_{0}$. In a random sample of lefttruncated single spells, the individual likelihood contribution equals $f_{u}(t \mid x) /(1-$ $\left.F_{u}\left(t_{0} \mid x\right)\right)$ with $F_{u}$ being the distribution function associated with the density $f_{u}$. With multiple spells per unit (or group or stratum), left-truncation of a spell duration outcome can be defined analogously, regardless of whether other spells are observed for this unit where the outcome exceeds its lower threshold. However, sometimes none of the duration outcomes of a unit is observed or used if at least one of them is left-truncated. The study of cause-specific mortality with twin data mentioned in Section 1 is such an example. For expositional reasons it is useful to 
consider this case first. If the number of spells (observed or not observed) of a unit is known then the model can be used to derive the likelihood function. Suppose that each unit consists of two spells $j=1,2$ and that the spells are observed conditional on both spell durations surviving up to their truncation points $t_{01}$ and $t_{02}$, respectively. This might be called "strong left-truncation". In the simple case of no censoring, the likelihood contribution $L$ of the unit is now given by the density function of $t_{1}, t_{2} \mid T_{1}>t_{01}, T_{2}>t_{02}, x$, which can be expressed as

$$
L=\int_{0}^{\infty} f_{c}\left(t_{1} \mid T_{1}>t_{01}, x_{1}, v\right) f_{c}\left(t_{2} \mid T_{2}>t_{02}, x_{2}, v\right) d G\left(v \mid T_{1}>t_{01}, T_{2}>t_{02}, x\right)
$$

with $x=\left(x_{1}, x_{2}\right)$ and $T_{j}$ denoting the random duration variables. We thus average over the conditional frailty distribution $G\left(v \mid T_{1}>t_{01}, T_{2}>t_{02}, x\right)$ in units where both spells survive up to their truncation points $t_{0 j}$ (and given $x$ ). This is distribution of $v$ in the sample of observed spells. It can be expressed in terms of the model primitives through

$$
d G\left(v \mid T_{1}>t_{01}, T_{2}>t_{02}, x\right)=\frac{\left(1-F_{c}\left(t_{01} \mid x_{1}, v\right)\right)\left(1-F_{c}\left(t_{02} \mid x_{2}, v\right)\right) d G(v)}{\int_{0}^{\infty}\left(1-F_{c}\left(t_{01} \mid x_{1}, w\right)\right)\left(1-F_{c}\left(t_{02} \mid x_{2}, w\right)\right) d G(w)}
$$

where

$$
1-F_{c}\left(t_{0 j} \mid x_{j}, v\right)=\exp \left(-\Lambda\left(t_{0 j}\right) \phi\left(x_{j}\right) v\right)
$$

Note that even if only one of the spells $j$ within a unit has $t_{0 j}>0$, the distribution $G\left(v \mid T_{1}>t_{01}, T_{2}>t_{02}, x\right)$ differs from $G(v)$.

Assuming a gamma-distributed frailty with $E(v)=1$ and $\operatorname{Var}(v)=\sigma^{2}$ yields $^{5}$

$L=\phi\left(x_{1}\right) \lambda\left(t_{1}\right) \phi\left(x_{2}\right) \lambda\left(t_{2}\right)\left(\sigma^{2}+1\right)\left(1+\sigma^{2} M\left(t_{01}, t_{02}\right)\right)^{1 / \sigma^{2}}\left(1+\sigma^{2} M\left(t_{1}, t_{2}\right)\right)^{-\left(1 / \sigma^{2}+2\right)}$,

where $M\left(t_{1}, t_{2}\right)=\phi\left(x_{1}\right) \Lambda\left(t_{1}\right)+\phi\left(x_{2}\right) \Lambda\left(t_{2}\right)$. Note that for ease of exposition we omit the dependence of $M$ on $x_{1}, x_{2}$.

Instead of the above type of left-truncation, we may consider sampling schemes with different types of reduced observability of low spell durations in a sharedfrailty model. If only one spell per unit is not left-truncated then one may nevertheless include it in the data used for inference. However, given that the number of spells per unit equals two, we directly infer that the other spell duration $t_{j}$ satisfies $t_{j} \leq t_{0 j}$. In other words, $t_{j}$ is left-censored instead of left-truncated. The unit then provides a likelihood contribution equal to $\int_{v} f\left(t_{1} \mid x_{1}, v\right) F\left(t_{2} \mid x_{2}, v\right) d G(v)$,

\footnotetext{
${ }^{5}$ See Appendix 1 for details.
} 
where we took $j=2$ and where $F_{c}$ denotes the cumulative distribution function of $t_{2} \mid x_{2}, v$.

Alternatively, the number of spells per unit may not be fixed and may increase with the sample size. Jensen et al. (2004) provide a detailed formal likelihood derivation in a rather general dynamic sampling framework where the number of (possibly simultaneously occurring) spells per unit may increase with the time that units are followed, and where all observed spells per unit are used for the statistical inference. Under some assumptions, the likelihood contributions are identical to equation (1). In particular, if two spells are observed for some unit, then the distribution of the frailty term of this unit, conditional on the two spell durations exceeding $t_{01}$ and $t_{02}$, respectively, equals $G\left(v \mid T_{1}>t_{01}, T_{2}>\right.$ $\left.t_{02}, x\right) .{ }^{6}$ Equation (2) replicates likelihood equations in e.g. Jensen et al. (2004) and Rondeau and Gonzalez (2005) for the shared gamma frailty model with lefttruncated data.

We now turn to the likelihood function used in Stata. The Stata Manual (e.g. Stata, 2009, p. 383) gives a likelihood contribution for the case of two possibly left-truncated spells and a shared gamma frailty model. This is used in the streg command with the options frailty(gamma) and shared(). In the absence of right-censoring, the likelihood contribution states that ${ }^{7}$

$$
L_{\text {Stata }}=\phi\left(x_{1}\right) \lambda\left(t_{1}\right) \phi\left(x_{2}\right) \lambda\left(t_{2}\right)\left(\sigma^{2}+1\right)\left(1+\sigma^{2}\left(M\left(t_{1}, t_{2}\right)-M\left(t_{01}, t_{02}\right)\right)\right)^{-\left(1 / \sigma^{2}+2\right)} .
$$

which obviously differs from equation (2). In Appendix 2 it is shown that the right hand side of equation (3) can be rewritten as

$$
L_{\text {Stata }}=\int_{0}^{\infty} f_{c}\left(t_{1} \mid T_{1}>t_{01}, x_{1}, v\right) f_{c}\left(t_{2} \mid T_{2}>t_{02}, x_{2}, v\right) d G(v)
$$

where $G(v)$ is a gamma distribution. This expression corresponds to the likelihood contribution presented in Gutierrez (2002, p.34) for general frailty distributions. By comparing equations (4) and (1) it is clear under which conditions equations (3) and (2) differ, and also what is the underlying reason for them to differ. First,

\footnotetext{
${ }^{6}$ Because of the dynamically evolving sampling scheme, where new spells per unit may start during the observation window, they need to make an approximation to deal with changes in the composition of the inflow during the observation window. This is an additional complication that does not affect the issues we focus on but which does not allow us to draw on their simulation results to assess the bias due to ignoring the interplay between left-truncation and dynamic selection.

${ }^{7}$ We translate the notation of the Stata Manual, as follows: $S_{i j}\left(t_{i j}\right)=e^{-\phi\left(x_{i j}\right) \Lambda\left(t_{i j}\right)}$ and $h_{i j}\left(t_{i j}\right)=\phi\left(x_{i j}\right) \lambda\left(t_{i j}\right)$, where we omit the index $i$.
} 
they differ if and only if $\operatorname{Var}(v)>0$ and at the same time at least one of the following inequalities applies: $t_{01}>0, t_{02}>0$. Secondly, they differ because the conditional densities in equation (4) are averaged over the inflow distribution $G(v)$ instead of the frailty distribution $G\left(v \mid T_{1}>t_{01}, T_{2}>t_{02}, x\right)$ conditional on the spell durations being left-truncated. The critical issue is that the likelihood in (3) treats the data as if no sorting had taken place prior to the beginning of the observation window. So it is implicitly assumed that the inflow distribution of frailties at $t=0$ does not change until the point of truncation. But since the subjects are at risk from $t=0$ onwards, this assumption cannot hold.

The above problem carries over to the case where the frailty is assumed to follow an Inverse-Gaussian frailty distribution in the streg command. The likelihood function for a shared frailty model with shared Inverse-Gaussian frailties and left-truncated duration data is derived in Appendix 3. This may be contrasted to the function given in the Stata Manual (Stata, 2009, p. 383).

An ad-hoc approach to deal with the discrepancy between the likelihood function and the Stata routine is to simply assume from the outset that the frailty distribution in the sample does not depend on $x$ and on the truncation points. This effectively amounts to a redefinition of the population, as the inflow into the state of interest at the moment of left-truncation, with the assumption that in this newly defined population, $v$ is independent of $x$ and of the elapsed time spent in the state of interest at the truncation point. Under this assumption, the Stata likelihood is correct. If the truncation points are not dispersed in the original population then such an approach may make sense. It replaces the assumption that $v$ and $x$ are independent in the inflow into the state of interest by the assumption that they are independent at the moment of truncation. If an MPH model guides the exit rate between the inflow and the truncation point, then the latter assumption in general entails that $x$ and $v$ are dependent in the original population that constitutes the inflow into the state of interest.

However, if the truncation points $t_{0 j}$ are dispersed then this approach does not make much sense. For example, consider two units $i, i^{\prime}$ each with two spells $j$. The units have identical systematic duration determinants including identical $x$ within and across units, but their left-truncation points differ. We take, in obvious notation, $0<t_{0 i 1}=t_{0 i 2}<t_{0 i^{\prime} 1}=t_{0 i^{\prime} 2}<\infty$, so that within each unit there is no dispersion of truncation points. The ad-hoc approach would require the distribution of $v$ in the first unit at $t_{0 i 1}$ to equal the distribution of $v$ in the second unit at $t_{0 i^{\prime} 1}$. But in the first unit, in between $t_{0 i 1}$ and $t_{0 i^{\prime} 1}$, the frailty distribution evolves dynamically over time in accordance to the shared frailty model, leading to a different distribution at $t_{0 i^{\prime} 1}$ than at $t_{0 i 1}$. By implication, the 
distributions of $v$ at $t_{0 i^{\prime} 1}$ would differ across units, not because the units behave differently, but because of the way in which they have been sampled.

So far, the Stata issues we discussed refer to the use of the options shared and frailty ( ) in the streg command, in conjunction with the use of the option enter () in the command stset. The streg command with the options shared and frailty() corresponds to parametric shared-frailty models. However, Stata also offers a routine for the semi-parametric estimation of shared-frailty models, and this routine can also be applied in the case of left-truncated data. Specifically, the stcox command with the options shared allows for the semi-parametric estimation of a shared-frailty model where $G(v)$ is assumed to be a gamma distribution, $\phi(x)=\exp \left(x^{\prime} \beta\right)$, and $\lambda(t)$ is an unspecified function (Cleves, Gould and Gutierrez, 2004). This command can be used in conjunction with the lefttruncation option enter () in the command stset.

The semi-parametric estimation method is developed by Therneau and Grambsch (2000) who do not discuss left-truncation of the duration data. It maximizes a penalized partial likelihood function, where the penalty function penalizes the distance between the fitted gamma distribution and the estimated frailty terms. Therneau and Grambsch (2000) show that with a particular choice of penalty function, this estimation method is equivalent to maximization of a full likelihood using an EM algorithm.

The Stata Manuals do not give likelihood expressions for the shared (gamma) frailty model in stcox if the data are left-truncated. This means that we do not know with certainty whether the command suffers from the same issue as the streg command. However, we can assess the performance of the command with simulated data. In the next section we show by way of simulations that most likely the stcox command does suffer from the same issue as the streg command. We should point out that, apart from the above, the stcox command with the shared option has the disadvantage that the reported standard errors of the estimated $\beta$ coefficients are under-estimated in that they are obtained under the assumption that the true variance of the gamma frailty distribution equals the estimated variance (Cleves, Gould and Gutierrez, 2004).

We finish this section by revisiting the cases where the Stata likelihood function and our own likelihood function coincide. Recall that if none of the spells is left-truncated then they coincide, and if there is no systematic unobserved heterogeneity ( $\operatorname{so} \operatorname{Var}(v)=0$ ) then they coincide as well. If a unit or group always consists of one single spell, then the Stata likelihood and our likelihood do not coincide, but our likelihood should then coincide to the likelihood of the MLE estimator for a single-spell MPH setting with left-truncated data. We know that 
the latter is correctly specified in Stata. By implication, with left-truncated data, the Stata estimator for the shared frailty model with a single spell per unit does not equal the Stata estimator for the corresponding MPH model with single-spell data. This is readily verified. In the latter case the frailty distribution conditions on survival until the truncation point whereas in the former case it does not.

According to Hirsch and Wienke (2011), none of the other software packages with estimation routines for shared-frailty models allows for left- truncation, with the exception of an R package called Frailtypack. This uses the semi-parametric Rondeau and Gonzales (2005) estimator which uses a full likelihood function that does take account of the interplay between dynamic selection and left-truncation (their estimator penalizes non-smoothness of the baseline hazard function $\lambda(t)$ ).

\section{Simulation results}

Recall that we are not primarily interested in small-sample properties of estimators but rather in the appropriate choice of likelihood function. The latter should be visible in estimates based on a large sample. We simulate data from a shared frailty model. The sample consists of units each comprising two spells with a shared gamma frailty. The baseline hazard $\lambda(t)$ follows either a Weibull specification $\left(\lambda(t)=\alpha t^{\alpha-1}\right)$ or a Gompertz specification $\left(\lambda(t)=e^{\alpha t}\right)$. Furthermore, $\phi(X)=e^{X \beta}$ with $X=(1 x)$ and $x$ being a single time-constant covariate.

In a first step, the covariate $x_{i j}$ is drawn from a standard normal distribution for each spell $j$ of unit $i$, and the frailty term $v_{i}$ is drawn from a gamma distribution with $E(v)=1$ and $\operatorname{Var}(v)=\sigma^{2}$ for each unit $i$. The unknown model parameters are $\beta \equiv\left(\beta_{0} \beta_{1}\right), \alpha$ and $\sigma^{2}$. These have the following possible values,

$$
\beta_{0}=0, \quad \beta_{1}=1, \quad \alpha=1, \quad \sigma^{2} \in\{0.5,1,2\} .
$$

so we run simulations for three different values of the variance $\sigma^{2}$ of the frailty distribution. These values are in line with those in the simulations in Jensen et al. (2004).

In a second step, for given covariates, frailty terms and parameter values, the durations $t_{i 1}$ and $t_{i 2}$ are drawn independently from the distributions $F_{c}\left(t_{j} \mid x_{i j} v_{j}\right)$, $j=1,2$, respectively. ${ }^{8}$. Next, we draw the left-truncation thresholds $t_{0 i 1}$ and $t_{0 i 2}$ from a uniform distribution with range $(0, b)$. All units with $t_{i 1} \leq t_{0 i 1}$ or $t_{i 2} \leq t_{0 i 2}$

\footnotetext{
${ }^{8}$ We use the following transformation of the variable $u$ drawn from a uniform distribution $U(0,1): t_{i j}=\alpha^{-1} \log \left(1-\alpha \log \left(1-u_{i j}\right)\left(e^{X_{i j} \beta} v_{i}\right)^{-1}\right)$ which is the inverse of the cumulative distribution function $F_{c}\left(t_{i j} \mid X_{i j}, v_{i}\right)=1-\exp \left(-e^{X_{i j} \beta} \alpha^{-1}\left(e^{\alpha t_{i j}}-1\right) v_{i}\right)$.
} 
are dropped. This way the sample only contains those units for which both spell durations exceed their left-truncation points. The fraction $c \in[0,1]$ of data that are dropped due to left-truncation can be fine-tuned by modifying $b$. Effectively, the sample size of 50,000 units is determined by the requirement that each of the spells of these units has a duration exceeding a left-truncation point. In fact, if the data are sampled from the model with the Weibull specification with $\alpha=1$ and if $\sigma^{2}$ is large, then the estimation of the parameters $\beta_{0}, \alpha$ is numerically cumbersome. ${ }^{9}$ This suggests that a larger sample is needed for reliable inference, but in the light of the computational burden we opt for the alternative of assuming that the researcher knows that $\beta_{0}=0$.

In the last step of the simulation procedure we use the stset and streg commands to estimate a shared frailty model in Stata,

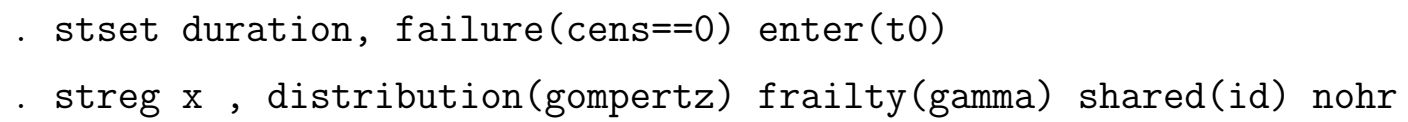

The results are summarized in Figures 1 and 2. The panels show the estimates of the constant $\beta_{0}$ (in the case of the Gompertz specification), the covariate effect $\beta_{1}$, the Gompertz duration dependence parameter $\alpha$, and the variance $\sigma^{2}$ of the gamma frailty distribution. We performed separate simulations with 30 different truncation rates $c \in[0,1)$, and we connect the resulting points to obtain the displayed curves.

All estimates move away from their true value as the truncation rate $c$ increases from zero. In particular, at any positive truncation rate, the covariate effect and the level of the hazard rate are under-estimated.

In general this is to be expected. As $c$ increases, the simulated distributions of $t_{0 i 1}$ and $t_{0 i 2}$ move to the right, so the difference between $G(v)$ and $G\left(v \mid T_{i 1}>\right.$ $\left.t_{0 i 1}, T_{i 2}>t_{0 i 2}, x\right)$ increases. Recall that $E(v)=1$, whereas with truncation, units with large $v$ will have exited the state relatively often before having reached the truncation point, so the mean of $v \mid T_{i 1}>t_{0 i 1}, T_{i 2}>t_{0 i 2}, x$ decreases in $t_{0 i j}$. The over-estimation of the mean frailty among the survivors at the truncation points is then compensated by an under-estimation of the magnitude of the other determinants of the level of the individual hazard rate (which by themselves have increasing effects on the individual hazard rate).

The bias towards zero of the estimate $\beta_{1}$ can be explained analogously. The true frailty distribution after truncation $G\left(v \mid T_{i 1}>t_{0 i 1}, T_{i 2}>t_{0 i 2}, x\right)$ depends

\footnotetext{
${ }^{9}$ More precisely, the estimation routine suffers from occasional numerical problems. This even occurs in the absence of left-truncation $(c=0)$ if $\sigma^{2} \geq 4$.
} 


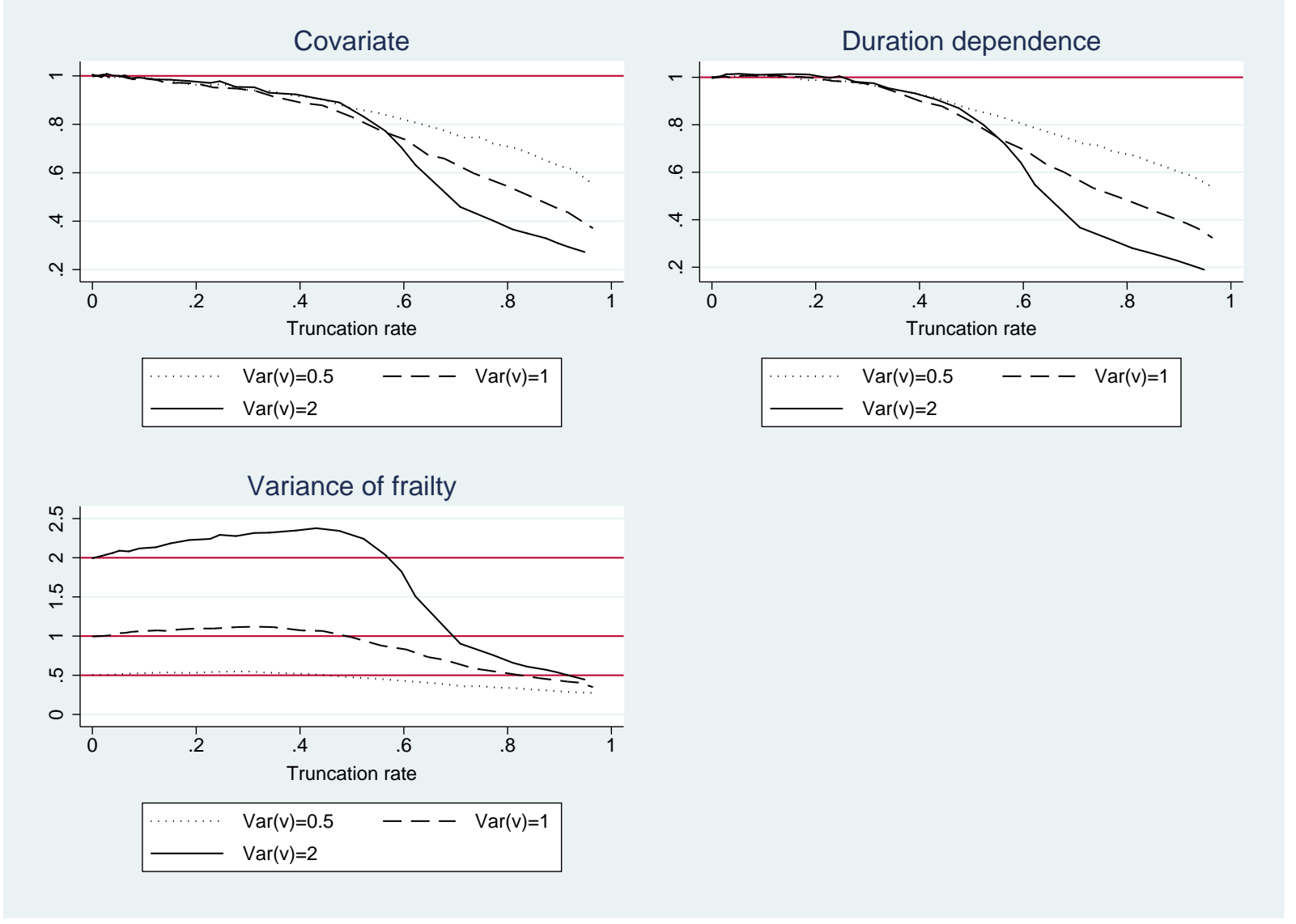

Figure 1: Simulation results of a shared gamma frailty model with Weibull duration dependence and left-truncated data using the Stata command streg with the option shared()

on the covariates $x$. Spells with a large value of $\exp \left(X_{i j} \beta\right.$ as well as a large $v_{i}$ terminate on average earlier than other spells. So in the case of a positive $\beta_{1}$, an observation in the truncated sample with a large $x$ is more likely to have a small $v_{i}$ than observations with low $x$. The association between $x$ and the observed hazard rates right after the truncation point is therefore smaller than $\beta_{1}$. If one ignores this, by ignoring the dynamic selection before the truncation point, then the resulting estimate of $\beta_{1}$ will be biased towards zero.

It may be instructive to consider some corresponding model expressions in the case of single-spell duration data. The correct expression for the observed hazard rate $\theta\left(t \mid T \geq t_{0}, x\right)$ at $t \geq t_{0}$ equals

$$
\theta\left(t \mid T \geq t_{0}, x\right)=\frac{\lambda(t) \exp \left(x^{\prime} \beta\right)}{1+\sigma^{2} \exp \left(x^{\prime} \beta\right) \Lambda(t)}
$$



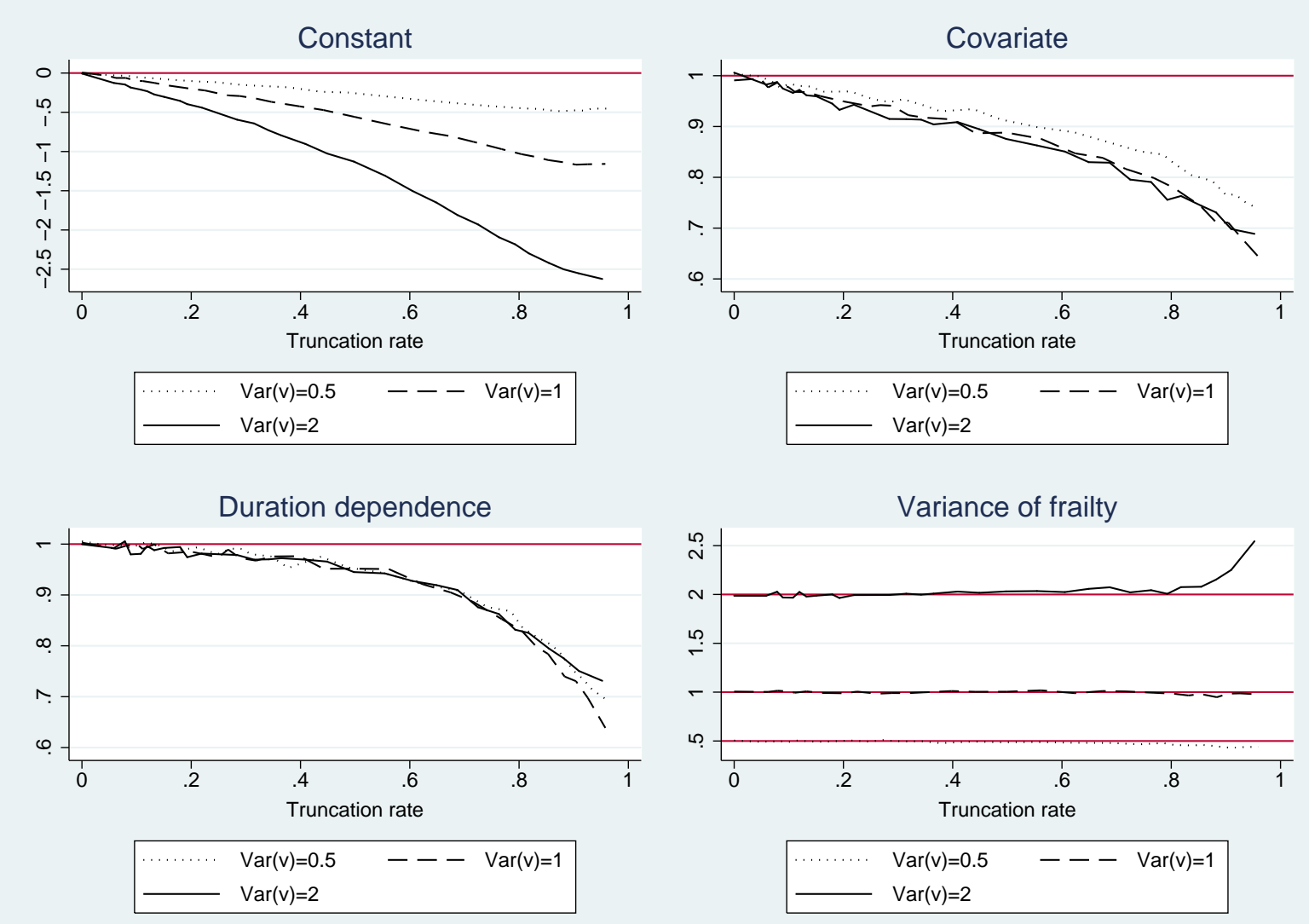

Figure 2: Simulation results of a shared gamma frailty model with Gompertz duration dependence and left-truncated data using the Stata command streg with the option shared()

This does not depend on $t_{0}$ because the hazard by definition conditions on $T \geq t$, which implies $T \geq t_{0}$. The expression for observed hazard assuming that there is no dynamic selection before $t_{0}$ is equal to

$$
\frac{\lambda(t) \exp \left(x^{\prime} \beta\right)}{1+\sigma^{2} \exp \left(x^{\prime} \beta\right)\left(\Lambda(t)-\Lambda\left(t_{0}\right)\right)}
$$

The estimates that follow from the latter approach lead to an estimated observed hazard at $t=t_{0}$ that fits the corresponding expression of (6) evaluated at the true parameter values. Hence,

$$
\widehat{\lambda}\left(t_{0}\right) \exp \left(x^{\prime} \widehat{\beta}\right)=\lambda\left(t_{0}\right) \exp \left(x^{\prime} \beta\right)\left(1+\sigma^{2} \exp \left(x^{\prime} \beta\right) \Lambda\left(t_{0}\right)\right)^{-1}
$$

For $t_{0}>0, \sigma>0$, this leads to the bias implications discussed above. 
Figures 1 and 2 also show that the bias of the estimates depends on the variance of the frailty distribution. As the latter increases, the estimates of the hazard level and the covariate effect move further away from their true values. Again, this is what would be expected. Notice that none of the biases vanishes for the sample size $n \rightarrow \infty$ for a given truncation rate.

It should be kept in mind that the simulation results in Figures 1 and 2 depend on the choice of baseline hazard and on the gamma frailty distribution as well as on the choice of the parameter values. For different models the magnitude of the bias may differ from the presented results.

For Stata users who wish to avoid misspecification of the likelihood function when estimating shared frailty models with left-truncated duration data, we programmed the Stata command stregshared, implementing the changes to the likelihood discussed in Section 2. In the appendix we give a short description of this new command. Simulations using stregshared confirm that the estimator is correct and that the estimates converge to their true values as $n \rightarrow \infty$ independent of the level of truncation.

As noted in the previous section, the Stata stcox command allows for semiparametric estimation of the shared gamma frailty model with left-truncated data. We use this routine to estimate this model with the simulated data. This does not impose the Weibull or Gompertz functional form for the duration dependence $\lambda$, and hence standard errors are larger than above. However, with our sample size, point estimates should be close to their asymptotic values. Instead, it turns out that the estimates are similar to those obtained with the appropriate streg command, for all values of $c$ considered. This confirms our conjecture that the stcox command in the case of the shared gamma frailty model with left-truncated data is programmed on the basis of the $L_{\text {Stata }}$ likelihood as defined in the previous section.

This result is of particular interest as the Stata stcox model has been frequently used in the empirical literature to estimate shared gamma frailty models, and sometimes the data are left-truncated. Gottard and Rampichini (2006) study the effects of poverty on time to childbirth among young women in Bolivia. In their data, individuals within a region are assumed to share their frailty term, and individuals are only included in their sample if they have reached at least the age of 14 at the time of the survey in 1998. Hence, left-truncation points vary across individuals. They state that they use the stcox, shared command in their empirical analysis. Studenski et al. (2011), who study the effect of gait speed on survival among elderly individuals, provide another example. They use data from 9 different cohort studies, and in a sensitivity analysis of their main results, they 
estimate shared gamma frailty models with Stata, where the frailty is taken to be cohort-study-specific. The individual lifetime durations are left-truncated by the entry age into the study. Hemmelgarn et al. (2007) study multidisciplinary care for elderly patients with chronic kidney disease and its effect on survival. They assume shared frailties for matched treated and untreated individuals, and they estimate shared frailty models with Stata and/or SAS. Their data are subject to left-truncation. Matching on age ensures that both lifetimes durations need to exceed a left-truncation point in order for the pair to be included in the sample.

\section{Conclusion}

This paper analyzes the implications of ignoring the effect of left-truncation of duration data on the distribution of unit-specific unobserved determinants in the sample, if multiple durations are observed per unit. In the presence of unobserved heterogeneity, it is vital to correctly account for the truncation that influences the composition of survivors in the sample, especially if the truncation thresholds vary across units.

Stata users estimating shared frailty models with the streg or stcox command need to be aware that with left-truncated data, the estimators of the covariate effects, the duration dependence and the variance of the frailty distribution may be inconsistent. The magnitude of the bias depends on the level of truncation and also on the variance of the frailty distribution of the data generating process. The good news is the fact that the parameter estimates for the covariate effects are typically biased towards zero. So in the worst case, effects have been underestimated by Stata. 


\section{References}

Abbring, J.H. and G.J. van den Berg (2007), "The unobserved heterogeneity distribution in duration analysis", Biometrika, 94, 87-99.

Chamberlain, G. (1985), "Heterogeneity, omitted variable bias, and duration dependence", in J.J. Heckman and B. Singer, editors, Longitudinal analysis of labor market data, Cambridge University Press, Cambridge.

Clayton, D. (1978), "A model for association in bivariate life tables and its application in epidemiological studies of familial tendency in chronic disease incidence", Biometrika, 65, 141-151.

Cleves, M.A., W.W. Gould and RG. Gutierrez (2004), An Introduction to Survival Analysis Using Stata, Stata Press, College Station.

Do, P. and S. Ma (2010), "Frailty model with spline estimated nonparametric hazard function", Statistica Sinica, 20, 561-580.

Gottard, A. and C. Rampichini (2006), "Shared frailty graphical survival models", Conference paper, International Conference on Statistical Latent Variables Models in the Health Sciences.

Guo, G. (1993), "Event-history analysis for left-truncated data", Sociological Methodology, 23, 217-243.

Gutierrez, R.G. (2002), "Parametric frailty and shared frailty survival models", The Stata Journal, 2, 22-44.

Hemmelgarn, B.R., B.J. Manns, J. Zhang, M. Tonelli, S. Klarenbach, M. Walsh et al. (2007), "Association between multidisciplinary care and survival for elderly patients with chronic kidney disease", Journal of the American Society of Nephrology, 18, 993-999.

Hirsch, K. and A. Wienke (2011), "Software for semiparametric shared gamma and log-normal frailty models: An overview", Computer Methods and Programs in Biomedicine, forthcoming.

Hougaard, P. (2000) Analysis of Multivariate Survival Data, Springer, Heidelberg.

Jensen, H., R. Brookmeyer, P. Aaby and P.K. Andersen (2004), "Shared frailty model for left-truncated multivariate survival data", Working paper, University of Copenhagen.

Kalbfleisch, J.D. and R.L. Prentice (1980), The Statistical Analysis of Failure Time Data, Wiley, New York. 
Lancaster, T. (1990), The Econometric Analysis of Transition Data, Cambridge University Press, Cambridge.

Nielsen, G.G., R.D. Gill, P.K. Andersen, and T.I.A. Sørensen (1992), "A counting process approach to maximum likelihood estimation in frailty models", Scandinavian Journal of Statistics, 19, 25-43.

Ridder, G. (1984), "The distribution of single-spell duration data", in G.R. Neumann and N. Westergård-Nielsen, editors, Studies in Labor Market Dynamics, Springer-Verlag, Heidelberg.

Ridder, G. and I. Tunalı (1999), "Stratified partial likelihood estimation", Journal of Econometrics, 92, 193-232.

Rondeau, V. and J.R. Gonzalez (2005), "Frailtypack: a computer program for the analysis of correlated failure time data using penalized likelihood estimation", Computational Methods and Programs in Biomedicine, 80, 154-164.

Stata (2009), Stata Survival Analysis and Epidemiological Tables, Reference Manual Release 11, Stata Press, College Station.

Studenski, S., S. Perera, K. Patel, C. Rosano, K. Faulkner, M. Inzitari et al. (2011), "Gait speed and survival in older adults", Journal of the American Medical Association, 305, 50-58.

Therneau, T.M. and P.M. Grambsch (2000), Modeling Survival Data, Springer, New York.

Van den Berg, G.J. (2001), "Duration models: specification, identification, and multiple durations", in: J.J. Heckman and E. Leamer (eds.), Handbook of Econometrics, Volume V, North-Holland, Amsterdam. 


\section{Appendix}

First, note that the gamma and Inverse-Gaussian distributions are both special cases of the non-negative exponential family with density

$$
f(v)=v^{\delta} e^{-\lambda v} m(v) \phi(\delta, \lambda)^{-1} .
$$

A shared frailty model with a frailty distribution of this family has the following survival function (see Hougaard, 2000):

$$
\begin{aligned}
S\left(t_{1}, t_{2} \mid x\right) & =\int_{0}^{\infty} v^{\delta} e^{-\left(\lambda+M\left(t_{1}, t_{2}\right)\right) v} m(v) d v \frac{1}{\phi(\delta, \lambda)} \\
& =\frac{\phi\left(\delta, \lambda+M\left(t_{1}, t_{2}\right)\right)}{\phi(\delta, \lambda)},
\end{aligned}
$$

with $M\left(t_{1}, t_{2}\right)=\phi\left(x_{1}\right) \Lambda\left(t_{1}\right)+\phi\left(x_{2}\right) \Lambda\left(t_{2}\right)$. The second equality follows from the fact that (8) is equivalent to $\phi(\delta, \lambda)=\int_{0}^{\infty} v^{\delta} e^{-\lambda v} m(v) d v$ and therefore $\phi(\delta, \lambda+$ $\left.M\left(t_{1}, t_{2}\right)\right)=\int_{0}^{\infty} v^{\delta} e^{-\left(\lambda+M\left(t_{1}, t_{2}\right)\right) v} m(v) d v$.

\section{A Gamma frailty}

Let us assume a gamma distributed frailty with $E(v)=1$ and $\operatorname{Var}(v)=\sigma^{2}$. This implies the following restrictions on the density function in (8)

$$
\delta=1 / \sigma^{2}-1, \quad \lambda=1 / \sigma^{2}, \quad m(v)=1, \quad \phi(\delta, \lambda)=\lambda^{-(\delta+1)} \Gamma(\delta+1),
$$

where $\Gamma\left(\sigma^{2}\right)$ is the gamma function. Substituting the expression for $\phi(\delta, \lambda)$ into the right hand side of equation (9) leads to

$$
\begin{aligned}
S\left(t_{1}, t_{2} \mid x\right) & =\frac{\left(1 / \sigma^{2}+M\left(t_{1}, t_{2}\right)\right)^{-1 / \sigma^{2}} \Gamma\left(1 / \sigma^{2}\right)}{1 / \sigma^{2-1 / \sigma^{2}} \Gamma\left(1 / \sigma^{2}\right)} \\
& =\left(1+\sigma^{2} M\left(t_{1}, t_{2}\right)\right)^{-1 / \sigma^{2}} .
\end{aligned}
$$

Since $f\left(t_{1}, t_{2} \mid x\right)=\frac{\partial^{2}\left(1-S\left(t_{1}, t_{2} \mid x\right)\right)}{\partial t_{1} \partial t_{2}}$ it follows

$$
f\left(t_{1}, t_{2} \mid x\right)=\frac{\partial M\left(t_{1}, t_{2}\right)}{\partial t_{1}} \frac{\partial M\left(t_{1}, t_{2}\right)}{\partial t_{2}}\left(\sigma^{2}+1\right)\left(1+\sigma^{2} M\left(t_{1}, t_{2}\right)\right)^{-\left(1 / \sigma^{2}+2\right)} .
$$

Finally, let us consider the likelihood contribution of a group $i$ comprising two subjects with truncation points $t_{01}$ and $t_{02}$ and no censoring. Combining the results from equation (11) and (12) leads to

$$
\begin{aligned}
& f\left(t_{1}, t_{2} \mid T_{1}>t_{01}, T_{2}>t_{02}, x\right)=\frac{f\left(t_{1}, t_{2} \mid x\right)}{S\left(t_{01}, t_{02} \mid x\right)} \\
& =\phi\left(x_{1}\right) \lambda\left(t_{1}\right) \phi\left(x_{2}\right) \lambda\left(t_{2}\right)\left(\sigma^{2}+1\right)\left(1+\sigma^{2} M\left(t_{01}, t_{02}\right)\right)^{1 / \sigma^{2}}\left(1+\sigma^{2} M\left(t_{1}, t_{2}\right)\right)^{-\left(1 / \sigma^{2}+2\right)}
\end{aligned}
$$

which is equation (2) from section 2. 


\section{B Likelihood in the Stata Manual}

The Stata Manual (Stata, 2009, p. 383) presents the following likelihood contribution for a group $i$ of a shared frailty model with a gamma frailty in the case of no censoring

$$
L=\phi\left(x_{1}\right) \lambda\left(t_{1}\right) \phi\left(x_{2}\right) \lambda\left(t_{2}\right)\left(\sigma^{2}+1\right)\left(1+\sigma^{2}\left(M\left(t_{1}, t_{2}\right)-M\left(t_{01}, t_{02}\right)\right)\right)^{-\left(1 / \sigma^{2}+2\right)} .
$$

Rearranging and choosing $\delta=1 / \sigma^{2}-1$ and $\lambda=1 / \sigma^{2}$ according to (10) yields

$$
L=\phi\left(x_{1}\right) \lambda\left(t_{1}\right) \phi\left(x_{2}\right) \lambda\left(t_{2}\right) \frac{\left(\lambda+M\left(t_{1}, t_{2}\right)-M\left(t_{01}, t_{02}\right)\right)^{-(\delta+3)} \Gamma(\delta+3)}{(\lambda)^{-(\delta+1)} \Gamma(\delta+1)} .
$$

Since we know that $\phi(\delta+2, \lambda+x)=(\lambda+x)^{-(\delta+3)} \Gamma(\delta+3)$ from (10) and that $\phi(\delta+2, \lambda+x)=\int_{0}^{\infty} v^{\delta+2} e^{-(\lambda+x) v} m(v) d v$ from equation (9) it follows

$$
L=\phi\left(x_{1}\right) \lambda\left(t_{1}\right) \phi\left(x_{2}\right) \lambda\left(t_{2}\right) \int_{0}^{\infty} v^{2} e^{-\left(M\left(t_{1}, t_{2}\right)-M\left(t_{01}, t_{02}\right)\right) v} \frac{v^{\delta} e^{-\lambda v} m(v)}{\lambda^{-(\delta+1)} \Gamma(\delta+1)} d v
$$

and once the restrictions (10) for the gamma distribution are imposed again

$$
L=\int_{0}^{\infty} f\left(t_{1}, t_{2} \mid T_{1}>t_{01}, T_{2}>t_{02}, x, v\right) d G(v) .
$$

\section{Inverse-Gaussian frailty}

Let us assume Inverse-Gaussian distributed frailty terms. Like with the gamma frailty, this imposes restrictions on the density in (8)

$$
\delta=-1 / 2, \quad m(v)=\psi^{1 / 2} \pi^{-1 / 2} e^{-\frac{\psi}{v}} v^{-1}, \quad \phi(-1 / 2, \lambda)=e^{-(4 \psi \lambda)^{1 / 2}} .
$$

Assuming $\psi=\lambda$ gives a mean frailty of 1 and choosing $\sigma^{2}=1 /(2 \lambda)$ yields $\operatorname{Var}(v)=\sigma^{2}$. Substituting the expression for $\phi(\delta, \lambda)$ into the right hand side of equation (9) leads to

$$
\begin{aligned}
S\left(t_{1}, t_{2} \mid x\right) & =\frac{\exp \left(-\left(4\left(\frac{1}{2 \sigma^{2}}\right)\left(\frac{1}{2 \sigma^{2}}+M\left(t_{1}, t_{2}\right)\right)\right)^{1 / 2}\right)}{\exp \left(-\left(4\left(\frac{1}{2 \sigma^{2}}\right)^{2}\right)^{\frac{1}{2}}\right)} \\
& =\exp \left(1 / \sigma^{2}-1 / \sigma^{2}\left(1+2 \sigma^{2} M\left(t_{1}, t_{2}\right)\right)^{1 / 2}\right) .
\end{aligned}
$$

Since $f\left(t_{1}, t_{2} \mid x\right)=\frac{\partial^{2}\left(1-S\left(t_{1}, t_{2} \mid x\right)\right)}{\partial t_{1} \partial t_{2}}$ it follows

$$
f\left(t_{1}, t_{2} \mid x\right)=\frac{\partial M\left(t_{1}, t_{2}\right)}{\partial t_{1}} \frac{\partial M\left(t_{1}, t_{2}\right)}{\partial t_{2}} \frac{\left(1+\sigma^{2}\left(1+2 \sigma^{2} M\left(t_{1}, t_{2}\right)\right)^{-\frac{1}{2}}\right) S\left(t_{1}, t_{2} \mid x\right)}{1+2 \sigma^{2} M\left(t_{1}, t_{2}\right)} .
$$


Finally, let us consider the likelihood contribution of a group $i$ comprising two subjects with truncation points $t_{01}$ and $t_{02}$ and no censoring. Combining the results from equation (13) and (14) leads to

$$
\begin{aligned}
& f\left(t_{1}, t_{2} \mid T_{1}>t_{01}, T_{2}>t_{02}, x\right)=\frac{f\left(t_{1}, t_{2} \mid x\right)}{S\left(t_{01}, t_{02} \mid x\right)} \\
& =\phi\left(x_{1}\right) \lambda\left(t_{1}\right) \phi\left(x_{2}\right) \lambda\left(t_{2}\right) \frac{\left(1+\sigma^{2}\left(1+2 \sigma^{2} M\left(t_{1}, t_{2}\right)\right)^{-\frac{1}{2}}\right) \exp \left(1 / \sigma^{2}-1 / \sigma^{2}\left(1+2 \sigma^{2} M\left(t_{1}, t_{2}\right)\right)^{1 / 2}\right)}{\left(1+2 \sigma^{2} M\left(t_{1}, t_{2}\right)\right) \exp \left(1 / \sigma^{2}-1 / \sigma^{2}\left(1+2 \sigma^{2} M\left(t_{01}, t_{02}\right)\right)^{1 / 2}\right)}
\end{aligned}
$$

\section{The command stregshared}

\section{D.1 Syntax}

The command stregshared (see http://www.ceee-mannheim.de) is designed as an alternative to streg when fitting a shared gamma frailty model to lefttruncated duration data. The size of the units over which the frailties are shared should not exceed two when using stregshared. The functional form of the baseline hazard can be specified as piecewise constant, Weibull, exponential or Gompertz. The command has a similar syntax to streg:

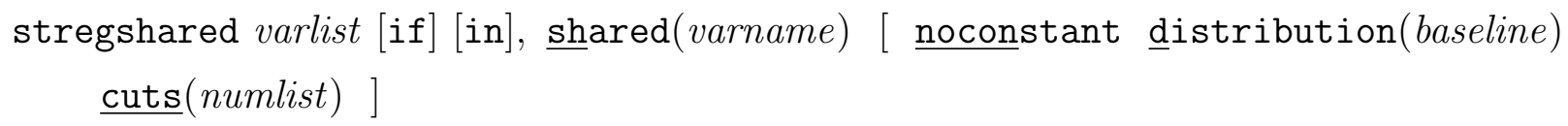

\section{D.2 Description}

stregshared is implemented as a v0 evaluator and uses Stata's modified NewtonRaphson maximization algorithm. The command fits the same shared frailty model as the streg command with the shared() option. The only difference is the adjusted likelihood function described in Section 2. Like streg it requires the data to be defined as duration data by stset and it uses the same variables in the same format as input arguments as streg.

\section{D.3 Options}

noconstant suppresses the constant term. The default is to include a constant in the model. Note that varlist should not include a constant term, when the option noconstant is not used.

distribution(baseline) sets the baseline hazard function to be of the type baseline, where baseline can be specified as weibull, exponential or gompertz. 
If this option is not used, a Weibull model is estimated. Note that the piecewise constant model requires this option to be specified as dexponential).

cuts(numlist) specifies the cutoff points of a piecewise constant baseline hazard. When the options noconstant and dexponential) are used, the option cuts (numlist) allows to estimate a piecewise constant model. Here, numlist holds the list of cutoff points, where the numbers have to be in strictly ascending order. For example, if the baseline function should be piecewise constant on the intervals $[0,5.5),[5.5,10)$ and $[10, \infty]$ use: nocon d (exponential) cuts $(5.5,10)$. The option cuts() can not be used with d(weibull) or d(gompertz).

shared(varname) specifies a variable defining the units within which the frailty is shared. The variable in varname is the same variable used in the option shared of streg. Recall that stregshared can only deal with a unit size of one or two spells. It is not a problem for the command if some (but not all) of the units have only one spell and others have two. But it cannot deal with units holding more than two spells. The shared() option has to be specified.

\section{D.4 Comparison to streg}

Since the stregshared command was designed as an alternative to streg, it is intended to work in a very similar way. So if one uses the original streg Stata command after stset to estimate a shared gamma-frailty model with a Weibull distribution

. stset duration, failure(fail $==1)$ enter(truncation)

. streg $x 1 \times 2 \times 3$, shared(id) d(weibull) frailty(gamma) nohr

the same arguments can be used with the stregshared command in order to estimate the same model with the adjustment in the likelihood function from Section 2:

- stset duration, failure(fail $==1)$ enter(truncation)

. stregshared $\mathrm{x} 1 \times 2 \times 3$, shared(id) d(weibull)

Here, id is the variable that identifies the unit. The same variable is used in the option shared() in streg. Note that the option nohr which causes streg to display the estimated parameter values instead of the hazard ratios is not used in our command. stregshared will display the parameter values as well as the 
hazard ratios in the estimation results.

In this example the data are left-truncated and therefore the enter (truncation) option in stset is used, where truncation is the variable that holds the lefttruncation points for each spell. If the enter() option is not used in stset, stregshared and streg will yield the same estimation results.

\section{D.5 Saved results}

When an estimation is run with stregshared, the command shows the choice of baseline function, the starting values, the number of units and total observations used in the estimation and finally the estimation results. These results include the parameter estimates, standard deviations, values of the test statistics and the hazard ratios.

stregshared saves the following in e():

Scalars :

est_base ancillary parameter (for Weibull or Gompertz function)

est_theta frailty parameter

Matrices :

est_b coefficient vector

estmatrix complete matrix of estimation results

(estimates, std. err. and test statistics)

To display the matrix of estimation results after running stregshared, type: matrix list e(est_matrix) 